\title{
Comparison of Profile and Sensitivity Patterns of Organism Causing Neonatal Sepsis between a Tertiary Care Neonatal Unit and DeNIS Study
}

\author{
Bharathi Balachander ${ }^{1}$, Gouthami Muktineni ${ }^{2}$, Suman Rao ${ }^{3}$
}

\begin{abstract}
Background: Infection is an important cause of mortality and morbidity in neonates. The rise in multidrug-resistant as reported by the Delhi Neonatal Infection Study (DeNIS) is alarmingly high. The aim of our study was to compare the results of the DeNIS with a single-center data from southern part of India

Materials and methods: This is a prospective observational study conducted between January 2016 and December 2017 in a tertiary care neonatal unit. Infants with blood culture positive sepsis were included in the study. The data were compared to the DeNIS study. A $p$ value of $<0.05$ was considered significant.

Results: The commonest organism identified in our study was CONS (40\%) followed by Klebsiella, E. coli, Candida, and non-fermenting gramnegative bacilli. The DeNIS collaboration identified Acinetobacter to be the commonest cause. Methicillin resistance prevailed in $70 \%$ of CONS and 50\% Staphylococcus aureus isolates in our study compared to $61 \%$ and $38 \%$ in the DeNIS. In the gram-negative organism, MDR was $66 \%$ for Acinetobacter, $15 \%$ for E. coli, and 44\% for Klebsiella. In the DeNIS, the rates of resistance were $82 \%, 54 \%$, and 38\% for Acinetobacter, E. coli, and Klebsiella, respectively. The mortality in MDRO was $26.9 \%$ compared to $15.7 \%$ in Denis.

Conclusion: Our findings conclude that the incidence of sepsis was similar to the DeNIS study, and antimicrobial resistance and the mortality secondary to the same is high. Unlike the DeNIS collaboration, the incidence of Acinetobacter was lower in our center. A nation-wide multicentric study on the profile of infection in neonates and antibiotic sensitivity is the need of the hour.

Keywords: Infection, Multidrug-resistant, Neonate.

Pediatric Infectious Disease (2020): 10.5005/jp-journals-10081-1258
\end{abstract}

\section{INTRODUCTION}

In neonates, sepsis is an important cause of morbidity and mortality. In developing countries, gram-negative organisms contribute to the major burden of neonatal sepsis. ${ }^{1}$ The knowledge on the prevalent profile of pathogens and their resistance patterns greatly aid clinicians in making empiric choices in antibiotics.

The Delhi Neonatal Infection Study (DeNIS) collaboration found a high incidence of sepsis and a great degree of antimicrobial resistance to infants born in tertiary-care centers and found Acinetobacter to be responsible for a large chunk of infections. ${ }^{2}$ The mortality rate was found to be high if there were associated with multidrug-resistance (MDR). The DeNIS study found no difference between early- and late-onset sepsis with respect to the causative organism. A subset of the study that analyzed data from the out born population concluded there was a high prevalence of MDR and fungal infections in this population. ${ }^{3}$

In high income, there are well-established multicentric infection surveillance networks. ${ }^{4,5}$ These networks are necessary, especially in countries like India, where culture, practices, language, and medical facilities widely varies from region to region. We aimed a retrospective analysis to compare infection profile at a tertiary-care institute in Bengaluru (which is a city in South India) to the DeNIS collaboration (from New Delhi in North India).

\section{Materials and Methods}

This study is an analysis of infection from the culture data and neonatal intensive care unit (NICU) database of St. John's Medical
${ }^{1-3}$ Department of Neonatology, St Johns Medical College Hospital, Bangaluru, Karnataka, India

Corresponding Author: Suman Rao, Department of Neonatology, St Johns Medical College Hospital, Bangaluru, Karnataka, India, Phone: +919019569652, e-mail: raosumanv@gmail.com

How to cite this article: Balachander B, Muktineni G, Rao S. Comparison of Profile and Sensitivity Patterns of Organism Causing Neonatal Sepsis between a Tertiary Care Neonatal Unit and DeNIS Study. Pediatr Inf Dis 2020;2(4):127-129.

Source of support: Nil

Conflict of interest: None

College Hospital over 2 years, January 2016 to December 2017. This is a 30-bedded tertiary-care center offering Level-3 Neonatal Intensive Care Unit (NICU) care. The NICU caters to inborn and out born infants.

All neonates with culture-positive sepsis were included in the study. The diagnosis and management of sepsis followed standard guidelines. Any clinical suspicion of sepsis or lab result positivity before 72 hours was termed as early-onset sepsis. After 72 hours, it was termed as late-onset sepsis. Neonates with risk factors for sepsis were labeled as suspect sepsis. Those with sepsis screen positive ( $C R P>1 \mathrm{mg} / \mathrm{dL}$, ANC - low for postnatal age). Neonates with culturepositive sepsis were labeled as definite sepsis. MDR was defined as resistance to three of five classes of antimicrobials. Lumbar puncture was performed in all infants with late-onset sepsis and any infant with unexplained central nervous system (CNS) symptoms. 
The culture-positive sepsis with organism sensitivity is prospectively collected every month, and it is deemed if the infection is healthcare associated or not. Daily data are collected to determine the denominator in the form of patient days, intravenous line days, intravenous fluid days, central line days, and ventilator days.

As a part of the National Neonatal-Perinatal Database, the total number of live births, NICU admissions, suspect, and probable sepsis data are collected. The data of NNPD was cross-checked by faculty month on month ensuring good quality of data.

Blood culture is collected using BACTEC systems. Blood culture is drawn before the initiation of antibiotics. At least, $1-2 \mathrm{~mL}$ of blood is inoculated into the blood culture bottle using the aseptic non-touch technique. Antibiotic sensitivity testing was done using VITEK systems.

A multipronged quality improvement initiative was started in 2016 with a target of zero CLABSI. ${ }^{6}$ The unit follows strict surveillance with a close response to outbreaks. ${ }^{7}$

\section{Statistical Analysis}

All continuous data were represented as mean \pm SD and analyzed using the $t$ test or Mann Whitney $U$ test. All categorical data were analyzed using the chi-square test. A $p$ value of $<0.05$ was considered significant.

\section{Results}

In the two years, there were 5,477 live births and 2,366 NICU admissions (Table 1). The incidence of total sepsis was $15.4 \%$

Table 1: Baseline characteristics of the study population

\begin{tabular}{llc}
\hline Parameter & DeNIS & Study site \\
\hline Total births & 88,636 & 5,477 \\
Total NICU admission & 13,530 (recruited) & 2,366 \\
Outborns & $?$ & 718 \\
Total sepsis & 1,934 & 356 \\
Culture positive & 840 & 150 \\
sepsis & & \\
\hline
\end{tabular}

compared to $14.3 \%$ in the DeNIS study. The commonest organism identified in our study was CONS (40\%) followed by Klebsiella, E. coli, Candida, and non-fermenting gram-negative bacilli. The DeNIS collaboration identified Acinetobacter to be the commonest cause. Methicillin resistance prevailed in $70 \%$ of CONS and $50 \%$ Staphylococcus aureus isolates in our study compared $61 \%$ and $38 \%$ in the DeNIS (Table 2). In the gram-negative organism, MDR was $66 \%$ for Acinetobacter, $15 \%$ for E. coli, and 44\% for Klebsiella. In the DeNIS, the rates of resistance were $82 \%, 54 \%$, and $38 \%$ for Acinetobacter, E. coli, and Klebsiella (Table 3). The mortality in MDRO was $26.9 \%$ compared to $15.7 \%$ in DeNIS.

\section{Discussion}

The DeNIS was one of the largest multicentric studies from India with 80,000 live births and 13,500 enrolled neonates. This was one of the first studies that involved prospective data collection, which enforced a good degree of methodological rigor. The data presented here involve a single-center data from a tertiary-care institute. $^{2}$ The prospective data surveillance is a routine part of the system following a quality improvement project to reduce central line-associated bloodstream infection.

Contrary to the DeNIS, the most common pathogen that was identified in our study was coagulase-negative Staphylococcus aureus. There were 18 infants with early-onset culture-positive sepsis. Gram-negative organisms (E. coli and Klebsiella) accounted for the majority of the pathogens in early-onset sepsis. The DeNIS reported that most of the infections occurred within 24 hours of life. In our series, only 18 of 58 culture positives were within 72 hours of life. In contrast to Western data, Group B Streptococcus was not found commonly (only 2 babies) in our study. However, it is being increasingly reported with increasing incidence in India. 8,9

Multidrug-resistance is an important problem that is being increasingly reported. The DeNIS study has reported a high degree of MDR in most of organisms that cause neonatal sepsis. These findings were also found in the Neo-AMR, a multicentric study that included both developed and developing countries. ${ }^{10}$ There was a high degree of MDR in gram-negative pathogens in our study as well.

Table 2: Organism isolated and their resistance patterns

\begin{tabular}{|c|c|c|c|c|c|}
\hline \multirow[b]{2}{*}{ Organism } & \multicolumn{2}{|r|}{ DeNIS } & \multicolumn{2}{|r|}{ Study site } & \multirow[b]{2}{*}{ pvalue } \\
\hline & Number & $M D R$ & Number & $M D R$ & \\
\hline \multicolumn{6}{|l|}{ Gram-negative sepsis } \\
\hline Klebsiella & 169 & $91(54 \%)$ & 18 & $8(44 \%)$ & 0.45 \\
\hline E. coli & 137 & $52(38 \%)$ & 13 & $2(15 \%)$ & 0.11 \\
\hline NFGNB & Nil & & 12 & $3(25 \%)$ & - \\
\hline Enterobacter & 44 & $22(50 \%)$ & 4 & $2(50 \%)$ & 1 \\
\hline Pseudomonas & 68 & $13(19 \%)$ & 4 & $1(25 \%)$ & 0.43 \\
\hline Acinetobacter & 222 & $181(82 \%)$ & 3 & $2(66 \%)$ & 0.51 \\
\hline Aeromonas & Not reported & & 2 & $0(0 \%)$ & - \\
\hline Citrobacter & & & 1 & $0(0 \%)$ & - \\
\hline \multicolumn{6}{|l|}{ Gram positive sepsis } \\
\hline Coagulase negative Staphylococcus aureus & 150 & 85 (MRSA) (61\%) 0 (VRSA) & 61 & 43 (MRSA) (70\%) 0 (VRSA) & 0.06 \\
\hline Staphylococcus aureus & 122 & 43 (MRSA) (38\%) 0 (VRSA) & 8 & 4 (MRSA) (50\%) 0 (VRSA) & 0.40 \\
\hline Enterococcus & 56 & 13 (VRE) (27\%) & 7 & 1 (VRE) (14\%) & 0.59 \\
\hline Beta hemolytic Streptococcus & Not reported & & 2 & 0 & - \\
\hline Alpha hemolytic Streptococcus & Not reported & & 3 & $1(33 \%)$ & - \\
\hline \multicolumn{6}{|l|}{ Fungal sepsis } \\
\hline Candida & Not reported & & 12 & 4 (flucon resistant) (33\%) & - \\
\hline
\end{tabular}


Table 3: Multidrug-resistant patterns in gram-positive and gramnegative isolates

\begin{tabular}{|c|c|c|}
\hline & Study site (\%) & DeNIS (\%) \\
\hline \multicolumn{3}{|c|}{ Gram-negative organisms } \\
\hline Cephalosporins & 68 & 47.9 \\
\hline Carbapenems & 22 & 45 \\
\hline MDR & 59.2 & 56.9 \\
\hline \multicolumn{3}{|c|}{ Gram-positive organisms } \\
\hline Methicillin & 26 & 37.7 \\
\hline Vancomycin & 6.6 & 9.3 \\
\hline
\end{tabular}

Although there is a high degree of MDR, the organism profile is different compared to the DeNIS study. This probably reflects that there may differences in bacteriological profiles in different parts of India.

The limitation of this study was that it was a single-center study, and the numbers were in no way comparable to the DeNIS study. However, the strength is the presence of prospective surveillance.

\section{ConcLusion}

Our findings conclude that the incidence of sepsis in our study was similar to the DeNIS study. Antimicrobial resistance and the mortality secondary to the same is also high. Unlike DeNIS collaboration, the incidence of Acinetobacter was lower in our center.

\section{IMPLICATIONS FOR RESEARCH}

A nation-wide multicentric study on the profile of infection in neonates and antibiotic sensitivity is the need of the hour.

\section{References}

1. Singhal N, National Neonatal-Perinatal Database. National Neonatal Perinatal Database Network: report for 2002-2003. http://www. newbornwhocc.org/pdf/nnpd_report_2002-03.PDF.

2. Investigators of the Delhi Neonatal Infection Study (DeNIS) collaboration. Characterization and antimicrobial resistance of sepsis pathogens in neonates born in tertiary care centers in Delhi, India: a cohort study. Lancet Glob Health 2016;4(10):e752-e760. DOI: 10.1016/ S2214-109X(16)30148-6.

3. Jajoo M, Manchanda V, Chaurasia S, et al. Alarming rates of antimicrobial resistance and fungal sepsis in outborn neonates in north India. PloS One 2018;13(6):e0180705. DOI: 10.1371/journal. pone. 0180705 .

4. Vergnano S, Menson E, Kennea N, et al. Neonatal infections in England: the NeonIN surveillance network. Arch Dis Child Fetal Neonatal Ed 2011;96(1):F9-F14. DOI: 10.1136/adc.2009.178798.

5. Leistner R, Piening B, Gastmeier P, et al. Nosocomial infections in very low birth weight infants in Germany: current data from the national surveillance system NEO-KISS. Klin Padiatr 2013;225(2):75-80. DOI: 10.1055/s-0033-1334886.

6. Balla KC, Rao SP, Arul C, et al. Decreasing central line-associated bloodstream infections through quality improvement initiative. Indian Pediatr 2018;55(9):753-756. DOI: 10.1007/s13312-018-1374-5.

7. Balachander $B$, Rajesh $D$, Pinhero $C L$, et al. Response measures to infection outbreaks during the second year of sustenance phase of infection control quality improvement. Indian J Pediatr 2020;87(5):333-338. DOI: 10.1007/s12098-020-03201-6.

8. Sridhar S, Grace R, Nithya PJ, et al. Group B streptococcal infection in a tertiary hospital in india-1998-2010. Pediatr Infect Dis J 2014;33(10):1091-1092. DOI: 10.1097/INF.0000000000000377.

9. Chaudhary M, Rench MA, Baker CJ, et al. Group B streptococcal colonization among pregnant women in Delhi, India. Pediatr Infect Dis J 2017;36(7):665-669. DOI: 10.1097/INF.0000000000001514.

10. Li G, Bielicki JA, Ahmed ASMNU, et al. Towards understanding global patterns of antimicrobial use and resistance in neonatal sepsis: insights from the NeoAMR network. Arch Dis Child 2020;105(1):26-31. DOI: 10.1136/archdischild-2019-316816. 